\title{
Study on measuring parameter lead of screw rotor based on CMM
}

\author{
Qiancheng Zhao ${ }^{\mathrm{a}}$, Tianlong Yang ${ }^{\mathrm{a}}$, Xiyun Yin ${ }^{\mathrm{a}}$, Zhongzhu Chen ${ }^{\mathrm{a}}$ \\ ${ }^{a}$ Hunan University of Science and Technology, Taoyuan Road, Xiangtan 411201, China \\ *Corresponding Author: eastlife0108@163.com
}

\begin{abstract}
Working surface of a screw rotor, the typical helical surface with complicated geometrical parameters, is hard to measure with CMM, especially parameter lead due to the difficulty of spiral scan and CMM probe compensation. Aiming at these problems, a new measurement method is proposed based on geometric feature models, expressing the helical surface and its offset surface separately. In this method, CMM probe compensation and spiral scan are no longer necessary, a cluster of uncompensated transverse section profiles in different axial positions are measured to obtain the parameter lead by calculating axial intercept and relative rotation angle. After performing the procedure of radial profile scan, rotary match and error evaluation, result shows that this method provides high precision.
\end{abstract}

Keywords: screw rotor, lead, CMM, offset surface.

\section{Introduction}

Twin-screw compressors are capable of high speed operation over a wide range of operating pressures and flow rates with high efficiencies, and two screw rotors, male and female of which, are the most important parts, when these are meshing with each other in opposite rotation direction, the gap between them reduces in size causing the air in the gap to be compressed ${ }^{(1)}$. The manufacturing quality of the two rotors directly influences the performance of a twin-screw compressor, so measurement of working surfaces, i.e., geometrical parameters of rotors play a big role in the course of rotor manufacturing. The geometrical parameters of a rotor include screw profile, lead, tip circle diameter, root circle, diameter, pitch diameter, rotor length and so on ${ }^{(2)}$. Among these parameters, lead is a more important parameter.

As shown in fig. 1 , two points, $a, b$, are the intersections of a spiral and two transverse section profiles of a rotor, $H$ is axial height difference, $\theta$ is relative rotation angle, lead can be expressed as :

$$
S=\frac{2 \pi}{\theta} H
$$

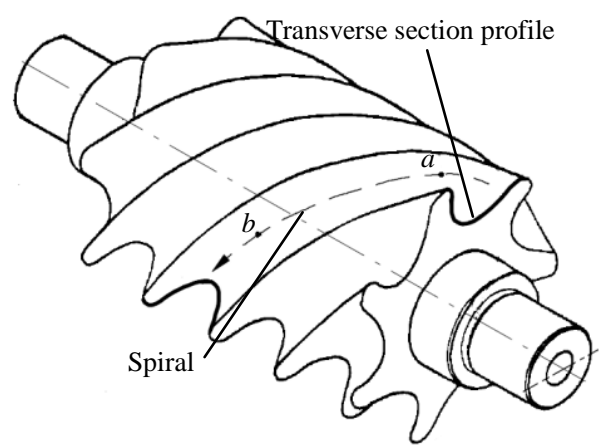

Fig. 1. Definition of parameter lead

In general, CMM is excellent for measuring geometrical parameters due to its high precision, but there are also some issues for measuring lead of rotors. On one hand, it's hard to operate CMM behavior by the means of measuring two points in a spiral without exact CAD model. On the other hand, because data directly obtained with CMM are coordinate values of probe centre, even if the corresponding points are contacted correctly, there will be large offset deviations between corrected and contacted points because of the difficulty of probe compensation for measuring complex helical surface ${ }^{(3)}$. Aiming at these problems, a method is proposed based on geometric feature models, expressing the helical surface and its offset surface separately to obtain lead simply by keeping measuring procedure from spiral scan and probe compensation.

\section{Principle}

As shown in fig. 2, a helical surface can be generated by spiral motion of a 2-D or 3-D curve, $L_{(t)}$, which doesn't overlap a spiral in this surface completely. Supposing centre axis of this helical surface overlaps $\mathrm{Z}$ axis of coordinate system, (O-XYZ), the equation with parameter $t$ for the 
curve is:

$$
L_{(t)}=\left[\begin{array}{lll}
x_{0(t)} & y_{0(t)} & z_{0(t)}
\end{array}\right]^{T}
$$

Then, the helical surface, $D_{(t, \theta)}$, can be defined as:

$$
D_{(t, \theta)}=\left[\begin{array}{c}
x_{0(t)} \cos \theta-y_{0(t)} \sin \theta \\
x_{0(t)} \sin \theta+y_{0(t)} \cos \theta \\
z_{0(t)}+\frac{\theta}{2 \pi} S
\end{array}\right]
$$

Where, $\theta$ is relative rotation angle, $S$ is lead. Therefore, relative rotation angle also can be calculated out by rotary match between the corresponding curves in the helical surface.

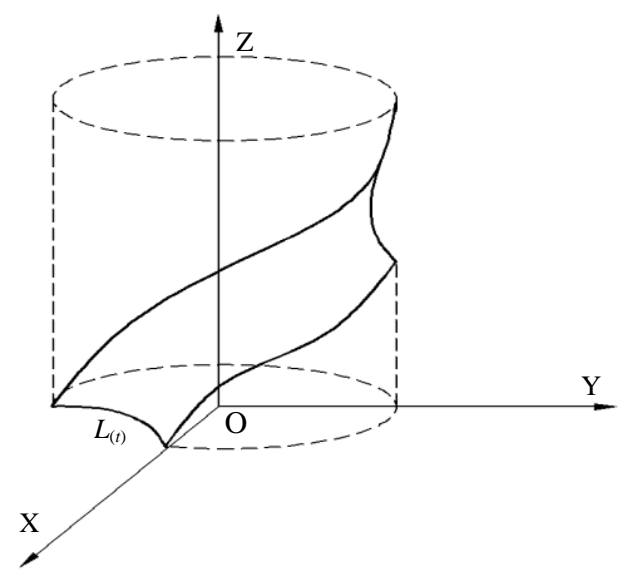

Fig. 2. Generation of screw surface

Supposing the equation with parameter $t$ and $\theta$ for the offset of $D_{(t, \theta)}$ is ${ }^{(4)}$ :

$$
\Delta_{(t, \theta)}=D_{(t, \theta)}+\rho \cdot n_{(t, \theta)}
$$

Where, $n_{(t, \theta)}$ is the common unit normal of a point in $D_{(t, \theta)}$ and the only corresponding one in $\Delta_{(t, \theta)}, \rho$ is the distance between $D_{(t, \theta)}$ and $\Delta_{(t, \theta)}$ in the direction of whichever common normal, based on formula $(3), n_{(t, \theta)}$ can be obtained as:

$$
n_{(t, \theta)}=H^{-1}\left[\begin{array}{c}
F_{1} \cos \theta+F_{2} \sin \theta \\
F_{1} \sin \theta-F_{2} \cos \theta \\
F_{3}
\end{array}\right]
$$

Where,

$$
\left\{\begin{array}{l}
{\left[\begin{array}{l}
F_{1} \\
F_{2} \\
F_{3}
\end{array}\right]=\left[\begin{array}{l}
\frac{S}{2 \pi} \frac{d y_{0(t)}}{d t}-x_{0} \frac{d z_{0(t)}}{d t} \\
\frac{S}{2 \pi} \frac{d x_{0(t)}}{d t}+y_{0} \frac{d z_{0(t)}}{d t} \\
x_{0} \frac{d x_{0(t)}}{d t}+y_{0} \frac{d y_{0(t)}}{d t}
\end{array}\right]} \\
H=\sqrt{F_{1}^{2}+F_{2}^{2}+F_{3}^{2}}
\end{array}\right.
$$

Then $\Delta_{(t, \theta)}$ can be transformed as:

$$
\Delta_{(t, \theta)}=\left[\begin{array}{c}
\left(x_{0(t)}+\rho \frac{F_{1}}{H}\right) \cos \theta-\left(y_{0(t)}-\rho \frac{F_{2}}{H}\right) \sin \theta \\
\left(x_{0(t)}+\rho \frac{F_{1}}{H}\right) \sin \theta+\left(y_{0(t)}-\rho \frac{F_{2}}{H}\right) \cos \theta \\
\left(z_{0(t)}+\rho \frac{F_{3}}{H}\right)+\frac{\theta}{2 \pi} S
\end{array}\right]
$$

Consequently, the offset of a helical surface is still helical and has a same parameter lead. Supposing working surface of a rotor is measured with CMM sequentially in the direction of centre axis, a new surface, the unit of probe centre paths, is an offset of the working surface because radius of probe tip is constant. Then, the parameter lead obtained by axial intercept and rotary match between probe centre paths, i.e., uncompensated transverse section profiles, also is suitable for the rotor.

\section{Profile Scanning}

As shown in fig. 3, scanning probe(model: SP600; manufactured by Renishaw Plc., UK) are installed on CMM(model: GLOBAL IMAGE 7107; manufactured by Brown \& Sharpe Inc., USA), a male rotor is fixed with a fixture to keep centre axis of which overlapping $\mathrm{Z}$ axis of measuring coordinate system. Radius of prober tip is selected to $1.5 \mathrm{~mm}$, scanning step length is set to $0.75 \mathrm{~mm}$, and Auto-Compensation built in CMM is closed, theoretical value of lead is $222.0000 \mathrm{~mm}$.

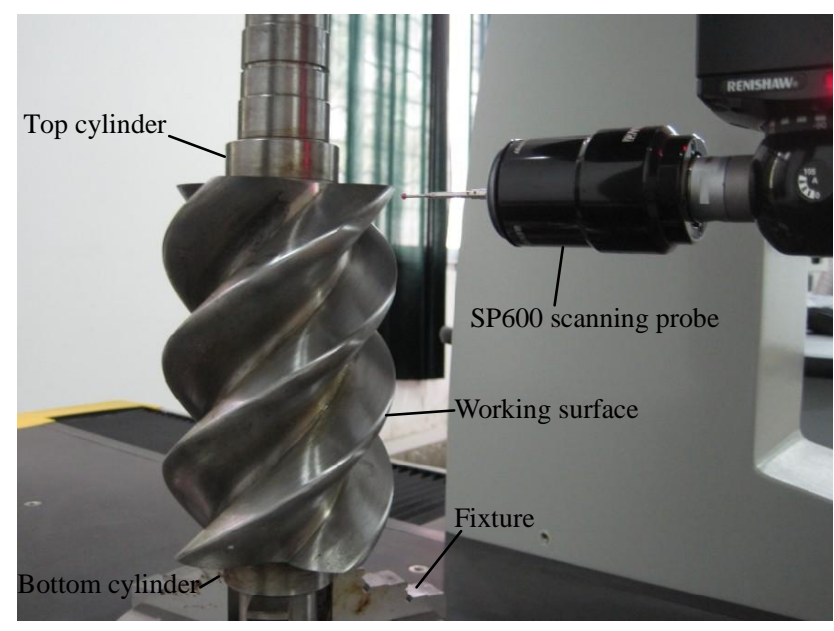

Fig. 3. Profile scanning

Steps are taken towards scanning a single tooth surface in the direction of centre axis to obtain a cluster of tooth profiles ${ }^{(5)}$, i.e., transverse section profiles, what's important, in the course of a single profile scanning, position $\mathrm{Z}$ of probe must be locked to keep the profile being radial, the top and bottom cylinders are also measured for obtaining 
coordinate values of centre axis. The completed point cloud of scanned data is shown in fig. 4 .

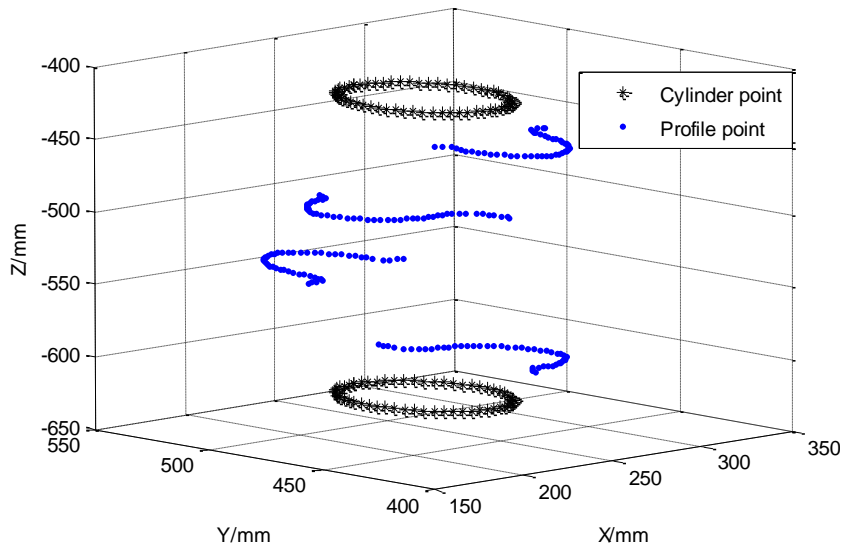

Fig. 4. Point cloud of profiles

\section{Data Processing}

\subsection{Profile fitting}

Center axes coordinates of top and bottom cylinders are calculated out by the means of the least square fit for coordinate translation, which causes centre axis of transverse section profiles overlapping $\mathrm{Z}$ axis of translated coordinate system. Cubic NURBS function ${ }^{(6)}$, as shown in formula (8), is set to construct continuous profile curves.

$$
p(u)=\frac{\sum_{i=0}^{n} w_{i} d_{i} N_{i, k}(u)}{\sum_{i=0}^{n} w_{i} N_{i, k}(u)}
$$

Where, $u$ is knot vector, $d_{i}$ is control points, $w_{i}$ is weight factors, and $N_{i, k}(u)$ is $k$ order basis functions, $k$ is set to 3 . We get a continuous curve of profile as shown in fig. 5 .

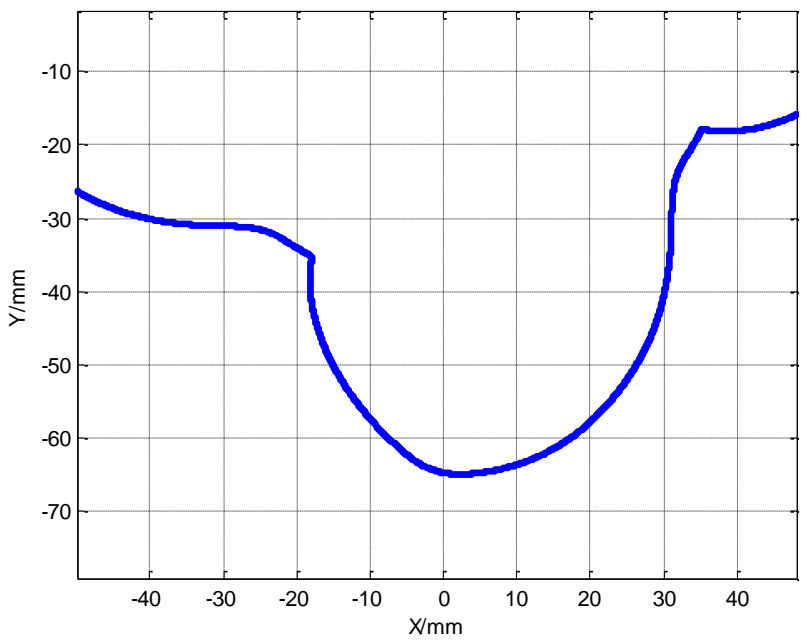

Fig. 5. Continuous curve of a rotor

\subsection{Rotary match}

Two continuous profile curves, $R$ and $I$, are defined as reference and target respectively. Before rotary match, initial rotation angle, $\theta$, is set to the angle between two radial vectors of the corresponding feature points, i.e., addendum or root points in $R$ and $I$. A certain amount of points, $\left[\begin{array}{ll}x_{i} & y_{i}\end{array}\right]$, are extracted from $I$ as sample, and then relative rotation angle is obtained by dichotomizing search, in the sequential loops, iterative functions are as follow:

$$
\left\{\begin{array}{l}
{\left[\begin{array}{ll}
\theta^{k} & \delta^{k}
\end{array}\right]=\left\{\begin{array}{ll}
{\left[\begin{array}{ll}
\theta & \delta
\end{array}\right]} & k=1 \\
\alpha_{\lambda_{k-1}^{k-1}}^{k} & \frac{\alpha_{\lambda_{k-1}+1}^{k-1}-\alpha_{\lambda_{k-1}-1}^{k-1}}{2}
\end{array}\right] \quad k \geq 2} \\
\alpha_{j}^{k}=\left[\begin{array}{ll}
\theta^{k} & \delta^{k}
\end{array}\right]\left[\begin{array}{ll}
1 & \frac{2 j-n-1}{n-1}
\end{array}\right]^{T} \\
I_{i, j}^{k}=\left[\begin{array}{ll}
x_{i} & y_{i}
\end{array}\right]\left[\begin{array}{ll}
\cos \alpha_{j}^{k} & -\sin \alpha_{j}^{k} \\
\sin \alpha_{j}^{k} & \cos \alpha_{j}^{k}
\end{array}\right] \\
D_{j}^{k}=\sum_{i=1}^{m} \min \left(\left|I_{i, j}^{k}-R\right|^{2}\right) \\
D_{\lambda_{k}}^{k}=\min \left(D_{j}^{k}\right) \quad \lambda_{k} \in(1,2,3 \ldots n) \\
\Delta^{k+1}=D_{\lambda_{k+1}}^{k+1}-D_{\lambda_{k}}^{k}
\end{array}\right.
$$

Where, $i=1,2,3 \ldots m, j=1,2,3 \ldots n$, are subdivision parameters of curve and angle respectively, $k=1,2,3 \ldots l$, is number of loops, $\delta$ is initial interval parameter. When $\Delta^{k+1}$ is smaller than the set threshold firstly, loop is interrupted to return the final relative rotation angle, $\theta^{k+2}$. The point cloud of a match procedure is shown in fig. 6. In the procedure of rotary match, the quantity and distribution of sample points directly influence the precision and speed, if possible, it is effective to obtain suitably larger quantity and more uniform distribution.

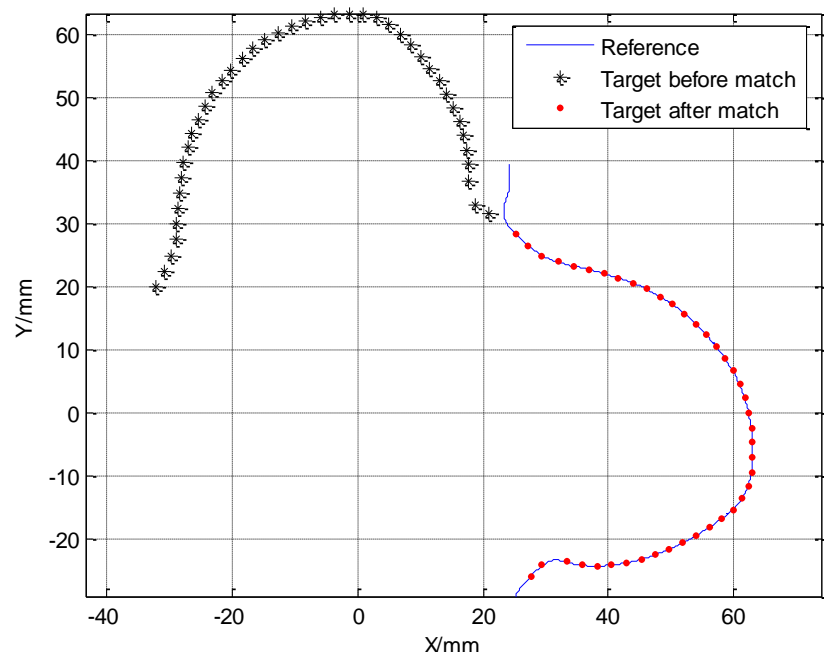

Fig. 6. Point cloud of rotary match

\subsection{Lead calculating}

After intercept in $\mathrm{Z}$ axis between reference and target is 
calculated out, lead can be obtained based on formula (1). The 6 individual values of lead corresponding to 6 groups of relative rotation angles and axial intercepts are shown in table 1.

Table 1. Calculation result of $H_{u, v}, \theta_{u, v}$ and $S_{u, v}$

\begin{tabular}{|c|c|c|c|}
\hline$u, v$ & $H_{u, v} / \mathrm{mm}$ & $\theta_{u, v} / \mathrm{rad}$ & $S_{u, v} / \mathrm{mm}$ \\
\hline 1,2 & 46.0000 & 1.3022 & 221.9570 \\
\hline 1,3 & 104.0000 & 2.9431 & 222.0293 \\
\hline 1,4 & 165.5000 & 4.6840 & 222.0019 \\
\hline 2,3 & 58.0000 & 1.6409 & 222.0840 \\
\hline 2,4 & 119.5000 & 3.3823 & 221.9932 \\
\hline 3,4 & 61.5000 & 1.7410 & 221.9497 \\
\hline
\end{tabular}

Supposing the standard deviations of relative rotation angles and axial intercepts are $s_{\theta}, s_{H}$, respectively, based on formula (1), the measurement error of lead can be expressed as formula (10) by the means of relative standard deviation.

$$
\frac{s_{S}}{S}=\sqrt{\left(\frac{\partial S}{\partial H} \frac{s_{H}}{S}\right)^{2}+\left(\frac{\partial S}{\partial \theta} \frac{s_{\theta}}{S}\right)^{2}}=\sqrt{\left(\frac{s_{H}}{H}\right)^{2}+\left(\frac{s_{\theta}}{\theta}\right)^{2}}
$$

Therefore, the beneficial conditions for decreasing the measurement error of lead are obtaining larger values of relative rotation angle and axial intercept, one among which changes against another by the means of strict linearity in theory. Then the equation for obtaining the final value of lead can be expressed as:

$$
\bar{S}=\frac{\sum S_{u, v} H_{u, v}}{\sum H_{u, v}}
$$

The final calculation result is $222.0042 \mathrm{~mm}$, referring to the theoretical value provided by manufacturer, $222.0000 \mathrm{~mm}$, the deviation is $4.2 \mu \mathrm{m}$.

\section{Conclusion}

A new method for measuring parameter lead of screw rotor based on CMM, in which CMM probe compensation and spiral scan are no longer necessary for obtaining parameter lead, measuring procedure is simplified as axial intercept and rotary match between the corresponding profiles scanned by scanning probe without radius compensation and result provides high precision. Theory and process discussed in this paper also provide the foundation for reconstructing complex helical surface and evaluating helical profile error.

\section{Acknowledgment}

This project is supported by National Nature Science Foundation of China (No:50875083, No:51275169), Hunan Provincial Natural Science Foundation of China (No:11JJ9016), Hunan Provincial Science and Technology Planning of China(No:2011FJ2018).

\section{References}

(1) Yu-Ren Wu, Zhang-Hua Fong : "Improved rotor profiling based on the arbitrary sealing line for twin-screw compressors", Mechanism and Machine Theory, Vol. 43, pp. 695-711, 2008

(2) Zhao Qiancheng, Lu Changyou, Xu Qiao, Huang Gen : "Analysing by Simulation to Evaluate the Method of Measuringn Srew rotors with CMM", Applied Mechanics and Materials, Vol. 36, pp. 64-65, 2010

(3) Brown\&Sharpe : "Quindos Scrcpm, Measurement of Screw Compressors”, Reference Manual, Wetzar, pp. 54-55, 1996

(4) ZHANG Li, TAN Jie-qing, LIU Zhi : "Rational Approximation of Offset Surface by Univariate S-Power Basis", Journal of Engineering Graphics, Vol. 1, pp. 104-109, 2010

(5) F.L. Litvin, P.H. Feng : "Computerized design, generation, and simulation of meshing of rotors of screw compressor", Mechanism and Machine Theory, Vol. 32, No. 2, pp. 137-160, 1997

(6) Yu Wuzhi, Hu Penghao, Zhao Qiancheng : "NURBS Models Buliding for Screw Rotor", Journal of Electronic Measurement and Instrument, Supplement, pp. 15-18, 2009 